\title{
A Standardized, Incremental Protocol to Increase Human Tolerance to the Cross-Coupled Illusion
}

\author{
Kathrine N. Bretl ${ }^{1 *}$, Sage O. Sherman ${ }^{1}$, Jordan B. Dixon ${ }^{1}$, Thomas R. Mitchell ${ }^{1}$, Torin K. Clark ${ }^{1}$
}

BACKGROUND: Humans can adapt to the "Coriolis" cross-coupled illusion with repeated exposure, improving the tolerability of faster spin rates and enabling short-radius, intermittent centrifugation for artificial gravity implementation.

OBJECTIVE: This investigation assesses the criticality of personalization in acclimation to the cross-coupled illusion.

METHODS: We used the median stimulus sequence of our previous effective and tolerable personalized, threshold-based protocol to develop a standardized (non-personalized) approach. During each of 10, 25-minute sessions, the spin rate was incremented independent of whether each subject reported experiencing the cross-coupled illusion.

RESULTS: In comparison to the previous personalized protocol, the standardized protocol resulted in significantly reduced acclimation to the cross-coupled illusion (17.7 RPM threshold for the personalized protocol versus 11.8 RPM threshold for the standardized) and generally increased motion sickness reports (average reporting of 1.08/20 (personalized) versus 1.98/20 (standardized)), on average. However, the lack of individualization also leads to significantly less variance in subjects' acclimation.

CONCLUSIONS: These findings are critical for future missions that may require several astronauts to be acclimated concurrently, due to resource and time constraints. Assessing feasibility of fast spin rate, short-radius centrifugation is crucial for the future of artificial gravity implementation during spaceflight.

Keywords: artificial gravity, short-radius centrifuge, physiological countermeasure, standardized acclimation

${ }^{1}$ University of Colorado at Boulder, 1111 Engineering Dr., Boulder, CO 80309

* Corresponding Author, 1111 Engineering Dr. Room 152, Boulder, CO 80309 (303) 492-4015, (303) 492-8883, kathrine.bret1@colorado.edu 


\section{BACKGROUND}

Artificial gravity (AG) has the potential to provide a comprehensive countermeasure for long-duration spaceflight. However, there currently exists a lack of optimized design for what is thought to be the most technologically and financially feasible approach for AG-the use of shortradius centrifugation [5,6]. This approach employs a centrifuge on the order of 4-8 meters in diameter, spinning at a rate of 15-30 rotations per minute (RPM). Historically, these rates have not been tolerable by humans, as fast spin rates cause the cross-coupled (CC) "Coriolis" illusion-a tilting or tumbling sensation experienced following the performance of an out-of-plane head tilt in a constantly rotating environment [10]. The CC illusion is very disorienting, often leading to motion sickness. Of relevance for short-radius centrifuge AG applications, the illusion is more intense when the subject is rotating at a faster spin rate [15]. This implies that the illusion becomes more provocative as the rotation radius (r) decreases, since the spin rate $(\omega)$ must increase to maintain the desired loading level $\left(g=a_{c}=\omega^{2} r, \omega=\sqrt{g_{\text {desired }} / r}\right)$. Early research suggested limiting the spin rate for AG applications to 4-6 RPM [8,9,12], effectively dismissing the feasibility of short-radius centrifuges due to how fast they would have to spin. To increase the utility of shorter-radius centrifuges that spin at a faster rate, humans must become more tolerant (i.e., acclimate) to the $\mathrm{CC}$ illusion.

Several decades of investigations have demonstrated the potential for human acclimation to the CC illusion with repeated exposure (e.g., reduced tilting and tumbling sensations) [14]. Typical acclimation protocols have used a constant, intense stimulus (i.e., exposing subjects to fast spin rates, typically $23 \mathrm{RPM})$. While effective in acclimating subjects, this acclimation comes at the cost of subject dropout rates of $25-35 \%$ due to severe motion sickness $[3,11,16,17]$. Subsequent research investigated the concept of incremental acclimation, in which spin rate was 
constant within the acclimation session but increased across days of the acclimation protocol [7] (14 RPM on day 1, 23 RPM on day 2, and 30 RPM on day 3), resulting in a dropout rate of only $14 \%$. This suggested the benefits of incremental acclimation, though the protocol was still too aggressive for some subjects. In an effort to accommodate all enrolled subjects, a more benign acclimation method was proposed. This method was both incremental within a session (rather than just increasing the exposure levels from one session to the next) and personalized (i.e., spin rate increased only when each subject did not experience the $\mathrm{CC}$ illusion) [4]. Across two consecutive days, this personalized, incremental approach had 0 of 10 subjects drop out due to motion sickness [4].

We recently extended this approach for a 10-day protocol in which the spin rate was incremented such that the CC illusion was always at or near each individual subject's threshold (i.e., where subjects reported not or just barely experiencing the illusion) at any given point in the study [2]. Subjects performed head tilts while spinning at a given spin rate. If a subject reported that no illusion was experienced following a pair of head tilts (i.e., head tilt down and head tilt back upright), the spin rate increased, otherwise it was maintained. This process continued for one 25-minute session per day for 10 subsequent days (each day the spin rate began at the fastest rate in which no illusion was reported on the previous day, starting at 1 RPM on the first day). Critically, the personalized, threshold-based protocol produced different stimuli for each individual subject (i.e., different spin rates across the 10-days), since the spin rate was maintained near each subject's CC illusion threshold. All 10 subjects were able to complete the full protocol with zero or very little motion sickness, and all subjects experienced an increase in $\mathrm{CC}$ illusion threshold (i.e., the fastest spin rate at which no illusion was experienced). Specifically, subjects increased their threshold from an average of 1.8 RPM at the beginning of the first session (range: 
1-3 RPM) to 17.7 at the end of the tenth and final session (range: 3-30 RPM). We note that unlike some of the earlier CC illusion acclimation studies, these subjects were not screened out for motion sickness susceptibility, and some of the subjects were even deemed highly susceptible based on their score on a pre-test questionnaire. The dramatic increase in spin rate at which no illusion was felt, coupled with little to no motion sickness and a $0 \%$ dropout rate, makes this an appealing approach to effectively acclimate all humans to the CC illusion (i.e., even those highly susceptible to motion sickness).

Multiple preceding studies have now highlighted the benefit, in terms of minimizing motion sickness, of a personalized and incremental acclimation protocol to improve tolerance of $\mathrm{CC}$ illusions. However, it remains unclear whether it is the incremental, low-spin-rate exposure or the threshold-based personalization (i.e., increasing spin rate based upon each individual's responses) that is critical for tolerability and efficacy, as all previous within-session incremental protocols (i.e., incrementally increasing spin rate during each session) have been personalized. Furthermore, operational constraints may not allow for the luxury of such an individualized approach. For example, future missions to the Moon or Mars may require all astronauts on board to be exposed to the same centrifugation levels concurrently. This would require the blanket approach of a non-personalized, or standardized, protocol to acclimate the entire crew to the CC illusion simultaneously.

\section{OBJECTIVE}

The objective of this investigation was to develop and test a standardized, incremental training protocol, then compare its efficacy and tolerability to that of the previously conducted personalized protocol. In doing so, we assessed the criticality of personalization in the acclimation

process. We hypothesized that the standardized protocol would still produce acclimation but 
would be less effective and less tolerable (i.e., induce more motion sickness) as compared to the personalized protocol.

\section{METHODS}

\subsection{Subjects}

In a between-subjects design, we compared two groups of subjects that completed either the previous personalized protocol [2] or the current standardized protocol. Both protocols were approved by the University of Colorado Institutional Review Board, and all subjects signed a written informed consent form. A total of ten healthy subjects (6 M/4 F), all university students, volunteered to participate in the standardized group, with an average age of 21.4 years (range: 1926). This was comparable with the personalized group (5M/5F, average 21.4 years, range: $18-24)$. In both groups, none of the subjects reported a history of vestibular dysfunction, and subjects were neither included nor excluded based on susceptibility to motion sickness. Each subject completed Reason and Brand's Motion Sickness Susceptibility Questionnaire (MSSQ) prior to their involvement in the study [13], and the standardized group scored between the 5th and 91st percentile with an average MSSQ score of 41.5 (SD: +/- 36.9). This was statistically consistent with the personalized group (average of $47, \mathrm{SD}:+/-29.3$, range: $10-99$, t-test, $\mathrm{p}=0.72$ ).

\subsection{Equipment and Procedure}

The equipment and procedure were identical to that used in our previous personalized study group ([2], Fig. 1). Experiments were performed in the dark to isolate vestibular cues and keep subjects naïve to the spin rate. Subjects were seated upright and spun about an Earth-vertical, yaw axis on the University of Colorado Boulder's Human Eccentric Rotator Device (HERD) for 25 minutes per day for 10 consecutive weekdays. Subjects made roll head tilts of 40 degrees in approximately one second, first from upright to right ear down, then back to upright. Head tilts 
were paced by the experimenter to ensure at least 30 seconds was provided between each action. Auxiliary equipment included two-way communication devices between the subjects and operators, wireless pushbuttons for redundant subject reporting of the CC illusion, infrared cameras for subject monitoring, and two foam blocks to ensure consistent head tilt angles.

\subsection{Standardized Protocol}

In the current standardized study group, all subjects were exposed to identical stimuli (i.e., spin rate increments and number of head tilts) for the first 9 sessions, then subjects completed a self-paced, personalized protocol for the $10^{\text {th }}$ and final session such that results could be compared with the previously completed personalized study. In every session (the first nine standardized), subjects were prompted when to make head tilts and were asked to report whether they experienced any $\mathrm{CC}$ illusion as a result of each head tilt. As in the personalized protocol, at the beginning of the first session, subjects performed one head tilt pair while spinning at 10 RPM to introduce the CC illusion with a pronounced, supra-threshold stimulus. During the standardized sessions, spin rate was incremented based on a pre-determined protocol regardless of subject reporting of the CC illusion (Figure 1 and table in Appendix). However, during the personalized session, spin rate was incrementally increased only when the subject reported feeling no illusion on both head tilts of one head tilt pair (tilt down and tilt back to the upright position).

The pre-determined standardized protocol was developed using median spin rates from the 10 subjects who completed the personalized protocol, sampled every 1 minute of testing. This approach created a standardized staircase for each of the 9 standardized sessions, aimed at producing an "average" of that experienced by the personalized group. An example session, Session 5, can be seen in Figure 1A. 
In calculating the staircase for the standardized protocol, we ensured that the protocol incremented by only 1 RPM during each step, that each commanded RPM was a whole number (a requirement based on HERD limitations), and that each RPM would be maintained for at least one minute such that one or more head tilt pair(s) could be achieved at each RPM. The resulting beginning and ending spin rates for each of the 9 standardized sessions can be seen in Figure 1B, with the corresponding full table of the sequence of spin rates and head tilts performed found in the Appendix.

The tenth and final session of the protocol employed the same personalized, incremental staircase that was used in our previous 10-day personalized investigation. In this session, the goal was to determine both the subject's individual beginning and ending thresholds as metrics to compare the efficacy between the personalized and standardized groups. As done previously [2], we defined the beginning and ending thresholds as the fastest spin rates at which no illusion was felt by the subject at the beginning and end of the session, respectively. In order to quantify the beginning threshold on the tenth session, we had to start spinning each subject at a spin rate below their threshold. If we spun the subject too fast and he/she felt the illusion on either head tilt of the first head tilt pair, we would be unable to know if the subject's beginning threshold was one RPM below their starting spin rate, or several RPMs below.

To avoid this, we created a decision tree to determine each subject's $10^{\text {th }}$-session starting spin rate. While appearing complex (Figure 2), this accomplished the simple objective of ensuring the starting spin rate on each standardized subject's $10^{\text {th }}$-session was below (but near) their threshold. In brief, the beginning threshold on the standardized session 9 could be used as the starting spin rate for session 10 (as in the personalized group), but only if it could be quantified using the standardized protocol (i.e., if the starting spin rate on session 9 of 6 RPM did not illicit 
an illusion, right arm of Figure 2). If this was in doubt for a subject based upon their responses on session 8 (i.e., they reported feeling the illusion at the starting spin rate of 6 RPM), a brief staircase was performed just before session 9 in order to determine an appropriate starting spin rate for session 10 (left arm of Figure 2). We were confident that the addition of this brief staircase (which was not necessary for the personalized subject group), would not have a measurable impact upon the performance of the standardized group. The staircase was designed to minimize the number of added head tilts, with at most three pairs, which is small compared to the $\sim 21$ head tilt pairs per session ( $\sim 190$ across sessions 1-9). Additionally, the staircase was designed to minimize the stimulus intensity of added head tilts by using lower spin rates first, where appropriate.

At each spin rate of the personalized session (session 10), subjects performed head tilts when prompted, just as they had in the previous 9 standardized sessions. However, in the personalized sessions, the spin rate incremented based on their responses to each pair of head tilts. At a given spin rate, if a subject reported that he/she did not feel the CC illusion on both the head tilt down and the head tilt back to upright, the spin rate was increased by 1 RPM over 20 seconds. If the subject reported that he/she did feel the CC illusion on either or both of the head tilts, the spin rate was maintained. In this way, the spin rate was incrementally increased based on subject reporting for the entirety of session 10. This was the exact protocol used for the personalized group. In both protocols, subject activity was uncontrolled and unmonitored between testing sessions; however, subjects were asked to avoid alcohol or excessive caffeine consumption within 12 hours before each testing session.

In addition to reporting if the subject felt the $\mathrm{CC}$ illusion on each head tilt of both the personalized and standardized sessions, we also asked the subjects to report their motion sickness rating (MSR) every 5 minutes of each 25-minute session, starting at minute five. This MSR was 
reported on a standardized scale of $0-20$, where 0 corresponded to absolutely no motion sickness symptoms, and 20 corresponded to extreme nausea or vomiting. We asked that subjects report at least an MSR of 1/20 if they were experiencing even the slightest motion sickness. The session concluded for the day if a subject reported an MSR of 10/20 or higher, and if this occurred on a second consecutive testing session, their participation in the study was concluded.

\subsection{Data Analysis}

\subsubsection{Dependent Variables}

As metrics quantifying the efficacy of the personalized and standardized acclimation protocols, we calculated the subjects' beginning and ending CC illusion thresholds. Specifically, we compared the threshold for the $10^{\text {th }}$ session of the standardized protocol group (the only session of the standardized protocol in which the spin rate was incremented based on subject feedback) to that for the $1^{\text {st }}$ session of the personalized protocol group (with no prior acclimation) to assess whether any acclimation had occurred over the 9 standardized sessions. Additionally, we compared the $10^{\text {th }}$ session of the standardized group to that for the $10^{\text {th }}$ session of the personalized protocol (with 9 previous sessions of threshold-based, personalized acclimation) to assess the efficacy of standardized vs. personalized protocols. To assess tolerability differences between the personalized and the standardized protocols, we were interested in the motion sickness levels reported by all subjects 1) across the first 9 sessions in both protocols and 2) in the $10^{\text {th }}$ session, where the protocol was personalized for both groups. These variables allow us to compare the protocols in terms of overall effectiveness, which includes the subjects' ability to acclimate to the CC illusion and with limited motion sickness.

\subsubsection{Statistical Tests}


Statistical tests were executed to compare the efficacy of the protocols. In all comparisons, Anderson-Darling and Shapiro-Wilks tests were used to assess the assumption of normality, and F-tests were used to assess equality of variance. If the data were normal, one-tailed, two-sample t-tests were run to compare groups. If two groups had significantly different variances, t-tests with unequal variance were used to compare means. If the data were not normal, one-tailed Wilcoxon rank sum tests were executed. We selected one-tailed tests as we a priori hypothesized that the standardized protocol would result in more acclimation than no training but would be less effective than the personalized protocol (i.e., personalized protocol would result in higher thresholds and lower motion sickness levels reported). All statistical tests were performed using MATLAB and R/RStudio.

\section{RESULTS}

Of the 10 subjects that were exposed to the standardized protocol, 9 of them completed the study. The final subject dropped out due to motion sickness after the $7^{\text {th }}$ session, when he/she reported an MSR of at least 10 for the second consecutive testing session. This subject scored in the $53^{\text {rd }}$ percentile for motion sickness susceptibility, and therefore, likely would not have been "screened out" due to a MSSQ cutoff (e.g., $90^{\text {th }}$ percentile score). Because this subject did not complete session 10 , he/she was not included in the statistical analysis, but his/her data is shown graphically (in gray) where applicable.

\subsection{Standardized Protocol Yielded Acclimation, but Less than in the Personalized Protocol}

\subsubsection{Beginning Thresholds}

The session 10 beginning thresholds achieved via the standardized protocol (white bar in Fig. 3A) averaged 5.0 RPM (95\% confidence interval: 1.9-8.1 RPM) but varied substantially between subjects (range $=0-13$ RPM, where a beginning threshold of 0 RPM corresponds to 
feeling the illusion at the minimum starting spin rate of 1 RPM). To determine if the standardized protocol resulted in any acclimation, we compared the beginning thresholds on the personalized session 1 (i.e., before these subjects were exposed to any CC illusion training) with those in the standardized session 10 (Wilcoxon rank sum, $\mathrm{W}=31.5, \mathrm{p}=0.14$ ). The beginning thresholds for the standardized session 10 showed a trend of acclimation (5.0 RPM) compared to untrained subjects (personalized session 1, 1.8 RPM), but the difference was not significant due to three subjects having a beginning threshold of zero (and personalized session 1 data not being normally distributed) as seen in Figure 3A.

Next, to evaluate if the first 9 days of the standardized protocol was as effective as the first 9 days of the personalized protocol, we compared the session 10 beginning thresholds of each protocol; the beginning thresholds on session 10 after the standardized protocol were significantly lower than the personalized protocol $(\mathrm{t}(17)=2.19$, personalized minus standardized diff $=7.6$ $R P M$, Cohen's $d_{\mathrm{rm}}=1.00, \mathrm{p}=0.02$ ). In fact, 5 of the 10 personalized subject's session 10 beginning thresholds were greater than all 9 of the standardized subjects'. We note that an F-test was conducted between the beginning thresholds of the session 10 personalized and session 10 standardized groups and found to be not significant (F-test, $F=4.002, p=0.06$ ), though trending towards higher variance in the personalized group.

\subsubsection{Ending Thresholds}

Following the standardized protocol, the ending threshold (i.e., the highest spin rate that yielded no perceived CC illusion at the end of the session) averaged 11.8 RPM (95\% confidence interval: 9.3-14.3 RPM). Again, we compared the ending threshold of session 10 of the standardized protocol to that of session 1 and session 10 of the personalized protocol (Fig. 3B). The standardized session 10 was found to have a significantly higher ending threshold than the 
personalized session $1\left(\mathrm{t}(17)=5.34\right.$, diff $=7.7$ RPM, Cohen's $\left.\mathrm{d}_{\mathrm{rm}}=2.45, \mathrm{p}<0.0005\right)$, but a significantly lower ending threshold than the personalized session 10 (unequal variance $t$-test, $t(17)$ $=-1.89$, diff $=-5.92$ RPM, Cohen's $\left.\mathrm{d}_{\mathrm{rm}}=-0.83, \mathrm{p}=0.04\right)$. The personalized protocol produced more acclimation as compared to the standardized protocol. However, for ending thresholds, the personalized protocol produced larger inter-subject variation than the standardized protocol (personalized SD: 9.1, standardized SD: 3.8, F-test, $F(9,8)=5.71, \mathrm{p}=0.02$ ). Thus, while standardization yielded less average acclimation, it was more consistent across subjects.

\subsection{Motion Sickness Generally Low, Yet Higher than Personalized Protocol}

Incremental in nature, the standardized acclimation protocol resulted in generally low motion sickness ratings across all subjects, yet only 9 of the 10 total subjects were able to complete the protocol. To quantify each subject's typical motion sickness level, we computed an average of their 45 MSRs reported across the 9 standardized sessions for each individual subject (Fig. 4A, which shows the subject that dropped out as a gray " $\mathrm{X}$ " but is not included in statistical tests). As compared to the personalized protocol, which yielded an MSR of 1.08/20 (95\% confidence interval: $0.35-1.82$ ), MSRs reported in the standardized approach were nearly twice as high, with an average of 1.98/20 (95\% confidence interval: 1.09-2.87); however this difference was not significant (Wilcoxon rank sum, $\mathrm{W}=63.5, \mathrm{p}=0.07$ ). Similarly, we found no statistically significant difference between protocols when comparing the average of each maximum reporting within the first 9 sessions ( $\mathrm{t}$-test, $\mathrm{t}(17)=1.46, \mathrm{p}=0.08$ ) (Fig. 4B). While these differences across the first 9 sessions were not significant, average MSRs on session 10 following the standardized protocol were significantly higher than those during the personalized session 10 (Wilcoxon rank sum, $\mathrm{W}=69.5, \mathrm{p}=0.02)($ Fig. $4 \mathrm{C})$. Thus, the standardized protocol was less effective at mitigating motion sickness on session 10, even though session 10 was incremented based upon each subject's 
response. In terms of the maximum motion sickness reported during session 10 (Fig. 4D), this difference was not significant (t-test, $\mathrm{t}(17)=1.32, \mathrm{p}=0.20$ ). Notably, in the standardized protocol most subjects experienced relatively low levels of motion sickness throughout testing (on the 0-20 scale).

Though not the primary focus of our study, several sets of Spearman rank order correlations were executed to identify if subjects who reported higher MSRs tended to acclimate faster or slower, or if their MSRs could be predicted by subjects' motion sickness susceptibility (MSSQ percentiles) reported prior to testing. Within the standardized protocol subjects, no significant correlations were found between: 1) average MSR across the first 9 sessions and session 10 ending and beginning thresholds ( $p=0.71$ for ending threshold, $p=0.74$ for beginning), 2 ) average MSR on session 10 and session 10 ending and beginning thresholds $(\mathrm{p}=0.60,0.93), 3)$ average MSR across the first 9 sessions and MSSQ $(\mathrm{p}=0.91)$, and 4) average MSR on session 10 and MSSQ (p $=0.72$ ). In addition, an exploratory investigation was completed to identify any potential correlations between subject response on head tilt pairs during the standardized sessions and subject's reported MSRs (i.e., did experiencing the CC illusion on most head tilt pairs cause more motion sickness?). Again, no significant correlations were found in any of the comparisons between: a) proportion of head tilts reported "yes, yes" or b) proportion of head tilts reported "no, no" in the standardized sessions, and: i) MSRs through session 9, ii) MSRs in session 10, or iii) MSRs averaged across all sessions $(\mathrm{p}=0.09-0.97)$. For these exploratory analyses, $\mathrm{p}$-values were not adjusted for the number of comparisons made.

\section{DISCUSSION}

\subsection{Efficacy of the Standardized Protocol}


The standardized protocol presented here was effective in acclimating individuals to the $\mathrm{CC}$ illusion, though to a lesser extent than the personalized protocol [2]. This conclusion can be reached in terms of both acclimation to the CC illusion (as determined by subjects' beginning and ending thresholds on the $10^{\text {th }}$ and final session of each protocol) and tolerability (as determined by subjects' reported motion sickness levels). As hypothesized, the personalized approach resulted in higher thresholds reached and lower motion sickness levels reported.

In the personalized protocol, we observed a large degree of inter-individual variability for both beginning and ending thresholds achieved in the final testing session. Because this protocol incremented the spin rate only when a subject did not feel the $\mathrm{CC}$ illusion, subject response dictated each subject's staircase. A smaller degree of inter-individual variability was observed in the final session of the standardized group, though this comparison only reached significance in the ending threshold metric. This suggests that a substantial portion of the inter-individual variability is due to the personalization of the incremental staircase across the first 9 sessions. In the standardized protocol subject group, only their final session was personalized, allowing for fewer differences in ending threshold acclimation to occur over the last 25-minute session. While this led to less interindividual variability, the standardized protocol may have prevented individuals from achieving higher spin rates, specifically those who would have otherwise been better (i.e., faster) acclimators.

Overall, MSRs were quite low in both protocols (recall a 20 corresponds to extreme motion sickness, near vomiting) and the dropout rate of $10 \%(1 / 10)$ for the standardized protocol is lower than that generally observed in previous acclimation studies of constant, high-intensity exposure.

We note that subjects for each study (both the personalized and standardized investigations) were recruited from the same subject pool. Subjects from both protocols shared similar distributions of age, gender, and motion sickness susceptibility (MSSQ percentiles). As 
such, we believe they can be directly compared to evaluate the efficacy of one protocol relative to the other.

\subsection{Implications for Future Artificial Gravity Applications}

The differences in CC illusion acclimation were both significant and operationally substantial. For example, the average beginning threshold for the personalized protocol on session 10 was 12.6 RPM, while that for the standardized group was only 5.0 RPM. In order to produce 1 Earth $\mathrm{G}\left(9.81 \mathrm{~m} / \mathrm{s}^{2}\right)$ of centripetal acceleration, for example, this increases the required centrifuge diameter from 11.3 to 71.6 meters, assuming the desired loading is administered at the riders' feet.

Alternatively, in order to acclimate to a desired spin rate based on centrifuge diameter, it would, on average, take longer with the standardized protocol than with the personalized. Our current study is unable to quantify the time course of acclimation for the standardized protocol, as the beginning and ending thresholds cannot be captured during sessions 1-9. A future study could further quantify this by having different subject groups complete a different number of standardized sessions (e.g., 1, 2, 4, 9, 14, 19 sessions), then assess the acclimation by quantifying beginning and ending thresholds in a final personalized session. However, existing datasets may be used to make some speculative extrapolations. First, the average acclimation across sessions appeared quite linear (increase in RPM per session) in the personalized protocol ([2], Fig. 4A\&B). If the acclimation rate is roughly linear for the standardized protocol as well, noting the beginning threshold for the personalized session 1 (no prior acclimation) averaged 1.7 RPM and the standardized session 10 averaged 5 RPM, then it would take approximately 29.7 sessions to reach the average beginning threshold observed on the personalized session 10 (12.6 RPM). In a similar analysis for the ending threshold, it would take approximately 15.8 sessions, thus the standardized 
protocol can be estimated to require $75-330 \%$ (15.8 or 29.7 divided by 9 ) times longer to acclimate to a desired spin rate.

In terms of motion sickness, the difference between personalized and standardized protocols, while significant in the session 10 average MSR comparison, could be considered slight. The average MSR (over the first 9 sessions) of the standardized group was nearly double that for the personalized group but was still relatively low ( 1.98 vs. 1.08 on a $0-20$ scale, where 20 corresponds to vomiting). In many operational scenarios, an average MSR of less than two would be considered sufficiently low and thus acceptable. On the other hand, one of our 10 standardized subjects was unable to complete the study due to motion sickness, as he/she reported an MSR $\geq 10$ on two consecutive sessions. In some scenarios (e.g., a crew of astronauts on their way to Mars) a dropout/excessive motion sickness prevalence of $10 \%$ would be unacceptable. Of course, this one subject may have just been abnormally susceptible (though their MSSQ scored in only the $53^{\text {rd }}$ percentile), highlighting the challenge of quantifying a low prevalence with a relatively small number of subjects $(\mathrm{N}=10)$. Future studies should better quantify the motion sickness dropout rate for the standardized protocol with a larger subject group.

Our results and implications have determined that the personalized protocol is superior in terms of tolerability and expedited acclimation for those with a predisposition to fast acclimation. However, this personalized protocol may not be feasible in future AG implementation in space. Personalization requires that each crewmember be spun individually for a specified amount of time. Limited crew time and resources may require that several crewmembers spin concurrently (i.e., at the same rate). If this were to be the case, this study has shown that it is likely that the crewmembers on-board would still be able to acclimate to the CC illusion, albeit more slowly. Personalization improves acclimation but does not appear to be a requirement. 
When applying these results to future AG applications, there are limitations which we have previously elaborated upon [2], but briefly list here. First, our subject pool happened to be younger than the current age demographic of NASA astronauts, which could alter CC illusion acclimation and/or associated motion sickness. Second, the Earth-vertical yaw rotation axis in our studies differs from typical centrifuge configurations, but still produces the unexpected stimulation to the semicircular canals when making a head tilt. Third, subjects were aligned with the rotation axis, so centripetal acceleration was not experienced, as would be in off-axis centrifugation. Finally, our ground-based study does not replicate the microgravity environment that astronauts experience while on orbit. We note, however, that our findings may be applicable in other domains that utilize the CC illusion outside of artificial gravity implementation [1].

\subsection{Different Approaches to Protocol Development}

In this study, we investigated only one method of standardization: using the median spin rates of the 10 subjects who completed the personalized protocol, sampled at every minute of testing. This was intended to provide a standardized protocol similar to what the "average" personalized subject had experienced. We expected this approach to be a reasonable first comparison to the personalized protocol, as we hypothesized it would be effective at inducing acclimation while still relatively tolerable in terms of motion sickness. However, there are many other approaches to the development of a generalized, standardized protocol.

Other methods we considered during the experimental design phase included using spin rates that represented either the $25^{\text {th }}$ or $75^{\text {th }}$ percentile (as opposed to $50^{\text {th }}$ percentile $=$ median) of the previously tested personalized protocol subjects. The $25^{\text {th }}$ percentile would be a less aggressive approach than the one we employed, and we expect that it would result in slower acclimation, yet less motion sickness and a lower subject dropout rate. Conversely, a $75^{\text {th }}$ percentile protocol would 
be more aggressive, potentially facilitating quicker acclimation at the cost of a potentially higher subject dropout rate. These hypotheses remain untested but offer the potential for developing "dose-response" curves for the effect of aggressiveness (in terms of percentile) of the standardized protocol on acclimation and motion sickness tolerability. Such an understanding would enable an optimal tradeoff between the two. Further, using the percentile of personalized protocol subjects to define the time course of the standardized staircase may not be ideal. One might imagine variants of a protocol that has the same beginning and ending spin rates, but either steps up with a fixed time increment across sessions, steps up less aggressively on early sessions and more aggressive on later sessions, or vice versa. Ultimately, the most effective protocol will depend on mission constraints and the ultimate goals and needs of the particular mission and crewmembers.

\section{CONCLUSIONS}

In this investigation, we have developed and tested an incremental, standardized protocol to acclimate humans to the $\mathrm{CC}$ illusion for future $\mathrm{AG}$ implementation. The protocol was developed to mimic the median stimulus of our previously published personalized protocol, in which spin rate was incremented only when subjects reported that they did not feel the CC illusion following a head tilt pair while spinning at a constant rate. The personalized incremental protocol resulted in a $100 \%$ completion rate, very low motion sickness, and dramatic acclimation to the $\mathrm{CC}$ illusion across 10 days. The purpose of this investigation was to evaluate the efficacy of an incremental, but standardized (i.e., non-personalized) protocol to determine the criticality of personalization in the acclimation process. We found that the standardized protocol was effective in acclimating subjects to the $\mathrm{CC}$ illusion, though significantly lower $\mathrm{CC}$ illusion thresholds were achieved as compared to the personalized protocol. Additionally, subjects in the standardized approach reported higher motion sickness ratings and only 9 of the 10 were able to complete the protocol. 
This investigation shows that personalization in CC illusion acclimation is beneficial, yet not required. If mission constraints required that all crewmembers be acclimated concurrently, it may take longer to reach a spin rate of operational interest (e.g., that required to apply $1 \mathrm{G}$ loading at the crewmembers' feet or heart, given the size of the centrifuge in use). The findings further suggest the tolerability and efficacy of using short-radius centrifugation for AG to comprehensively protect astronauts from spaceflight physiological deconditioning, helping enable future long-duration space exploration. 


\section{ACKNOWLEDGEMENTS}

This work was supported by a NASA Space Technology Research Fellowship. Preliminary results were presented at NASA's Human Research Program Investigators' Workshop in 2018.

\section{REFERENCES}

[1] R. Banks, D. Salisbury, P. Ceresia, The Canadian Forces Airsickness Rehabilitation Program, 1981-1991, Aviation, Space, and Environmental Medicine. 63 (1992) 1098-1101.

[2] K.N. Bretl, A.T. McCusker, S.O. Sherman, T.R. Mitchell, J.B. Dixon, T.K. Clark, Tolerable Acclimation to the Cross-Coupled Illusion through a 10-day, Incremental, Personalized Protocol, Journal of Vestibular Research, Accepted. (2019).

[3] E.L. Brown, H. Hecht, L.R. Young, Sensorimotor aspects of high-speed artificial gravity: I. Sensory conflict in vestibular adaptation, Journal of Vestibular Research. 12 (2002) 271282.

[4] C.C. Cheung, H. Hecht, T. Jarchow, L.R. Young, Threshold-based vestibular adaptation to cross-coupled canal stimulation, Journal of Vestibular Research. 17 (2007) 171-181.

[5] G. Clément, Human Research Program Human Health Countermeasures Element Artificial Gravity Evidence Report, NASA Lyndon B. Johnson Space Center, Houston, TX, 2015.

[6] G. Clément, A. Bukley, W. Paloski, Artificial Gravity as a Countermeasure for Mitigating Physiological Deconditioning during Long-Duration Space Missions, Frontiers in Systems Neuroscience. 9 (2015).

[7] P. Elias, T. Jarchow, L.R. Young, Incremental adaptation to yaw head turns during 30 RPM centrifugation, Experimental Brain Research. 189 (2008) 269-277. 
[8] A. Graybiel, R.S. Kennedy, E.C. Knoblock, F.E. Guedry, W. Mertz, M.E. McLeod, J.K. Colehour, E.F. Miller, A.R. Fregly, Effects of exposure to a rotating environment (10 RPM) on four aviators for a period of twelve days, Aerospace Medicine. (1965) 733-754.

[9] F.E. Guedry, R.S. Kennedy, C.S. Harris, A. Graybiel, Human performance during two weeks in a room rotating at three rpm, Aerospace Medicine. (1964) 1071-1082.

[10] F.E. Guedry, E.K. Montague, Quantitative Evaluation of the Vestibular Coriolis Reaction, Aerospace Medicine. 32 (1961) 487-500.

[11] H. Hecht, E.L. Brown, L.R. Young, Adapting to artificial gravity (AG) at high rotational speeds, in: Life in Space for Life on Earth, Stockholm, Sweden, 2002.

[12] R.S. Kennedy, A. Graybiel, Symptomatology during prolonged exposure in a constantly rotating environment at a velocity of one revolution per minute, Aerospace Medicine. (1962) $817-825$.

[13] J.T. Reason, J.J. Brand, Motion Sickness, Academic Press London, 1975.

[14] J.T. Reason, A. Graybiel, Progressive adaptation to coriolis accelerations associated with 1-rpm increments in the velocity of the slow rotation room, National Aeronautics and Space Administration, Naval Aerospace Medical Institute, Pensacola, Florida, 1969.

[15] S.E. Sheehan, L.R. Young, T. Jarchow, The effect of head turn velocity on cross-coupled stimulation during centrifugation, Journal of Vestibular Research. 18 (2008) 1-14.

[16] L.R. Young, H. Hecht, L.E. Lyne, K. Sienko, C.C. Cheung, J. Kavelaars, Artificial gravity: Head movements during short-radius centrifugation, Acta Astronautica. 49 (2001) 215-226. 
[17] L.R. Young, K.H. Sienko, L.E. Lyne, H. Hecht, A. Natapoff, Adaptation of the vestibuloocular reflex, subjective tilt, and motion sickness to head movements during short-radius centrifugation, Journal of Vestibular Research. 13 (2003) 65-77. 


\section{FIGURE CAPTIONS}
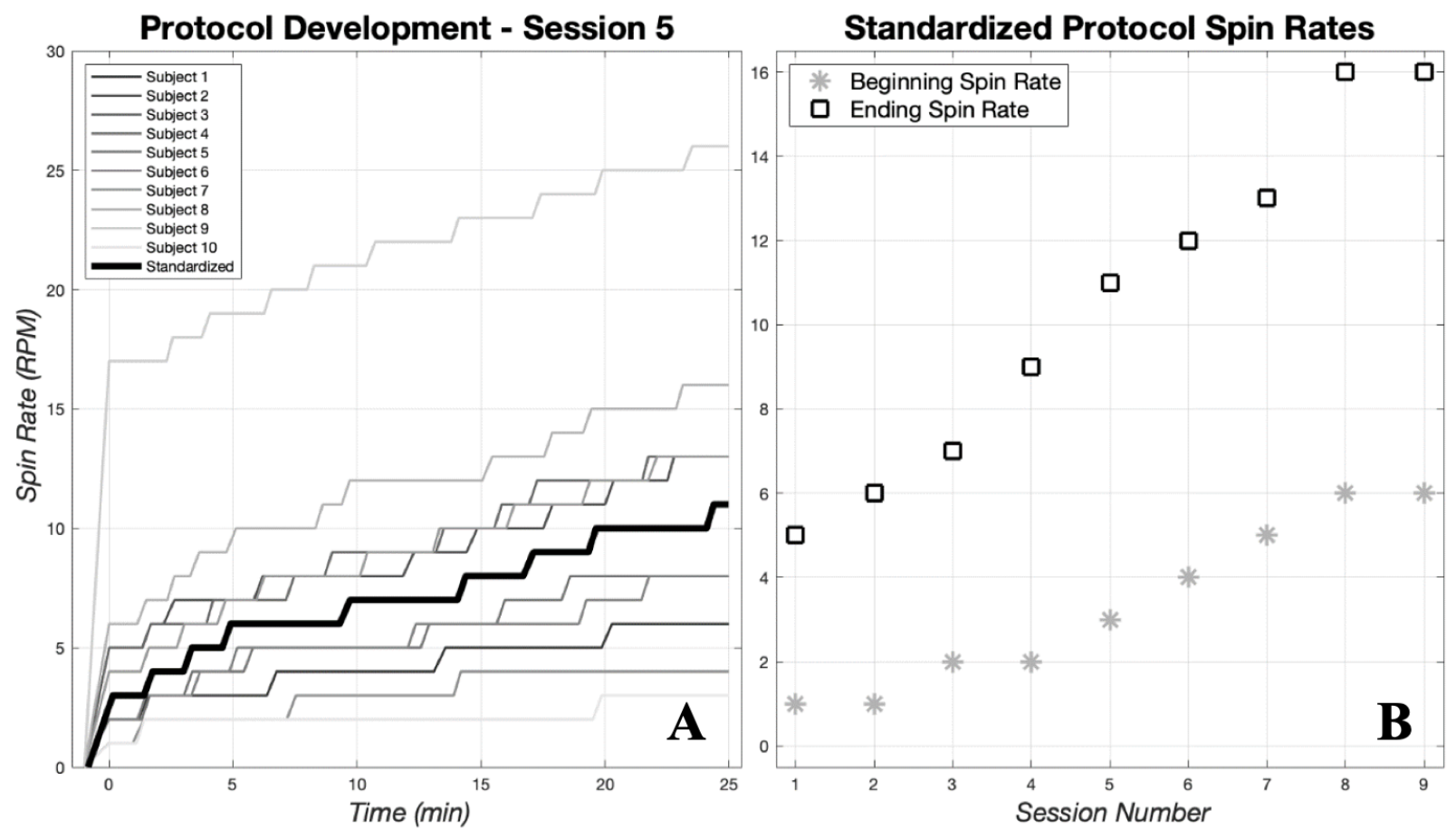

Fig. 1: A) Session 5 standardized protocol development. The thick black line shows the standardized staircase developed from calculating the median spin rates of the staircases from the 10 personalized subjects, depicted in various shades of gray. B) Beginning (asterisks) and ending (squares) spin rates of the first nine sessions of the standardized protocol. A unique incremental staircase was used to increase the speed from the beginning spin rate to the ending spin rate on each session (see table in Appendix). 


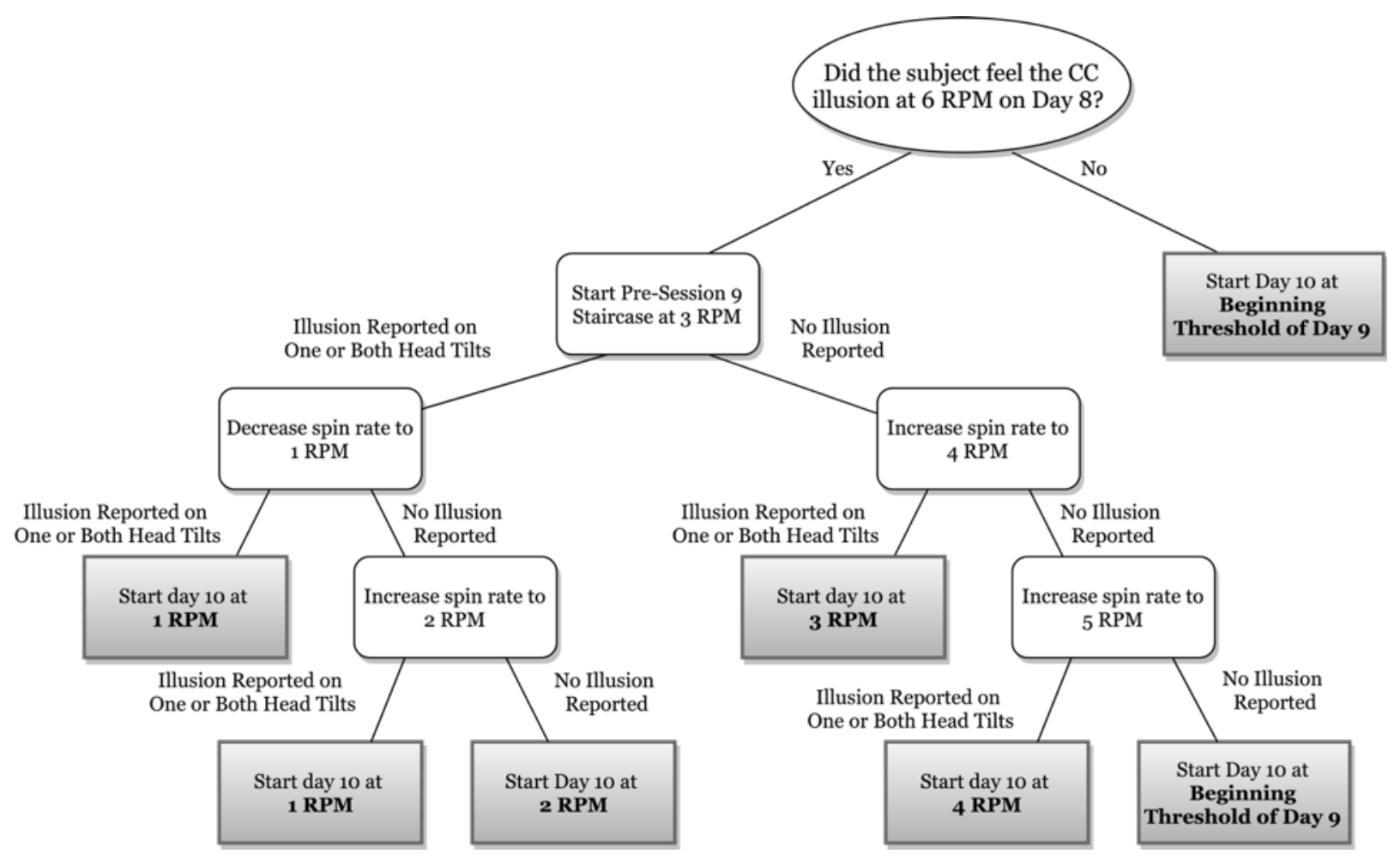

Fig. 2: Decision tree to determine how fast each subject should start spinning for the personalized testing session (session 10). 

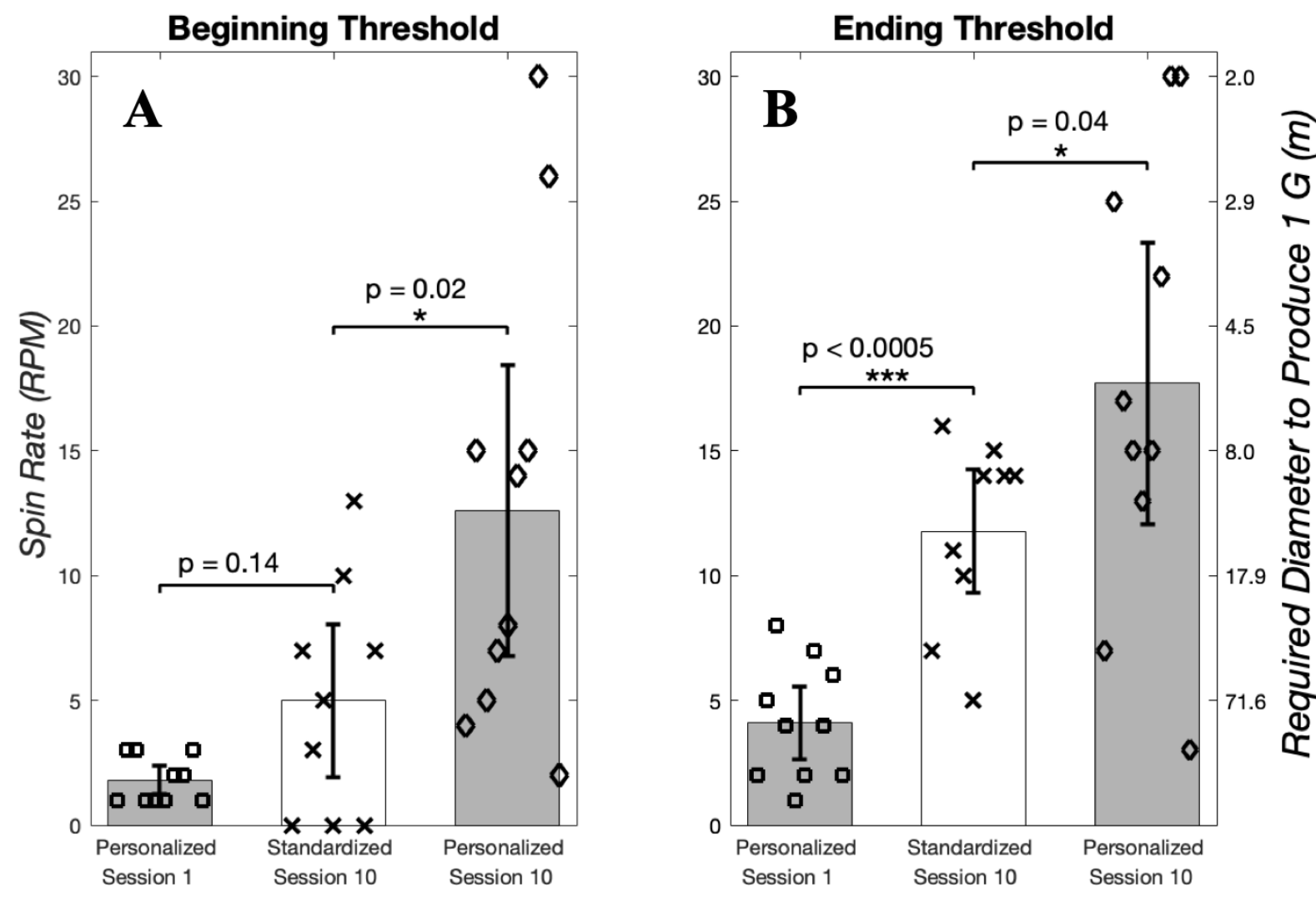

Fig. 3: Comparisons between personalized protocol session 1, standardized protocol session 10, and personalized protocol session 10 for Beginning (A) and Ending (B) thresholds. Each marker shows individual subjects, and the bars show averages with a 95\% confidence interval. Three of the four comparisons were statistically significant $\left(^{*}\right)$ when one-tailed t-tests were performed. For reference, the required centrifuge diameter to produce 1 Earth $G$ (at the rider's feet) for each spin rate is shown on the right y-axis (e.g., 10 RPM requires a $17.9 \mathrm{~m}$ diameter centrifuge to produce 1 G). 

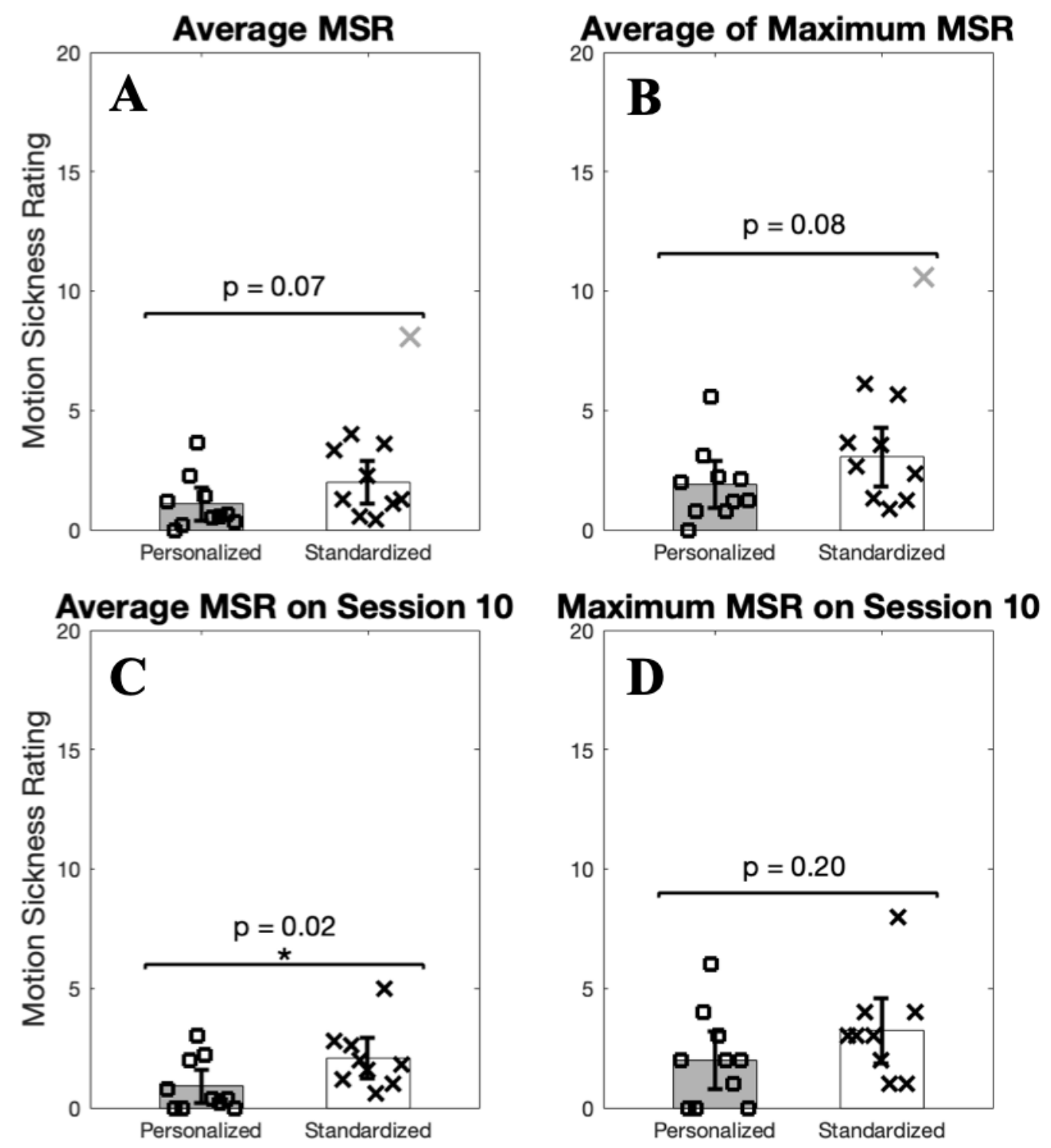

Fig. 4: Motion sickness rating comparisons between the personalized and standardized protocols. Each marker shows individual subject statistics for A) the average MSR across the first 9 sessions, B) the average of the maximum MSR reported in each of the first 9 sessions, C) the average MSR on the 10th session, and D) the maximum MSR on the $10^{\text {th }}$ session. In all plots, the bar shows the protocol average with a $95 \%$ confidence interval. Where applicable, a gray " $\mathrm{X}$ " shows the reports of the subject who dropped out due to motion sickness. His/her data was not 
included in the statistical analysis. Though the average standardized MSRs were significantly higher $(*)$ in the comparisons shown in C), they were still generally quite low. 


\section{APPENDIX}

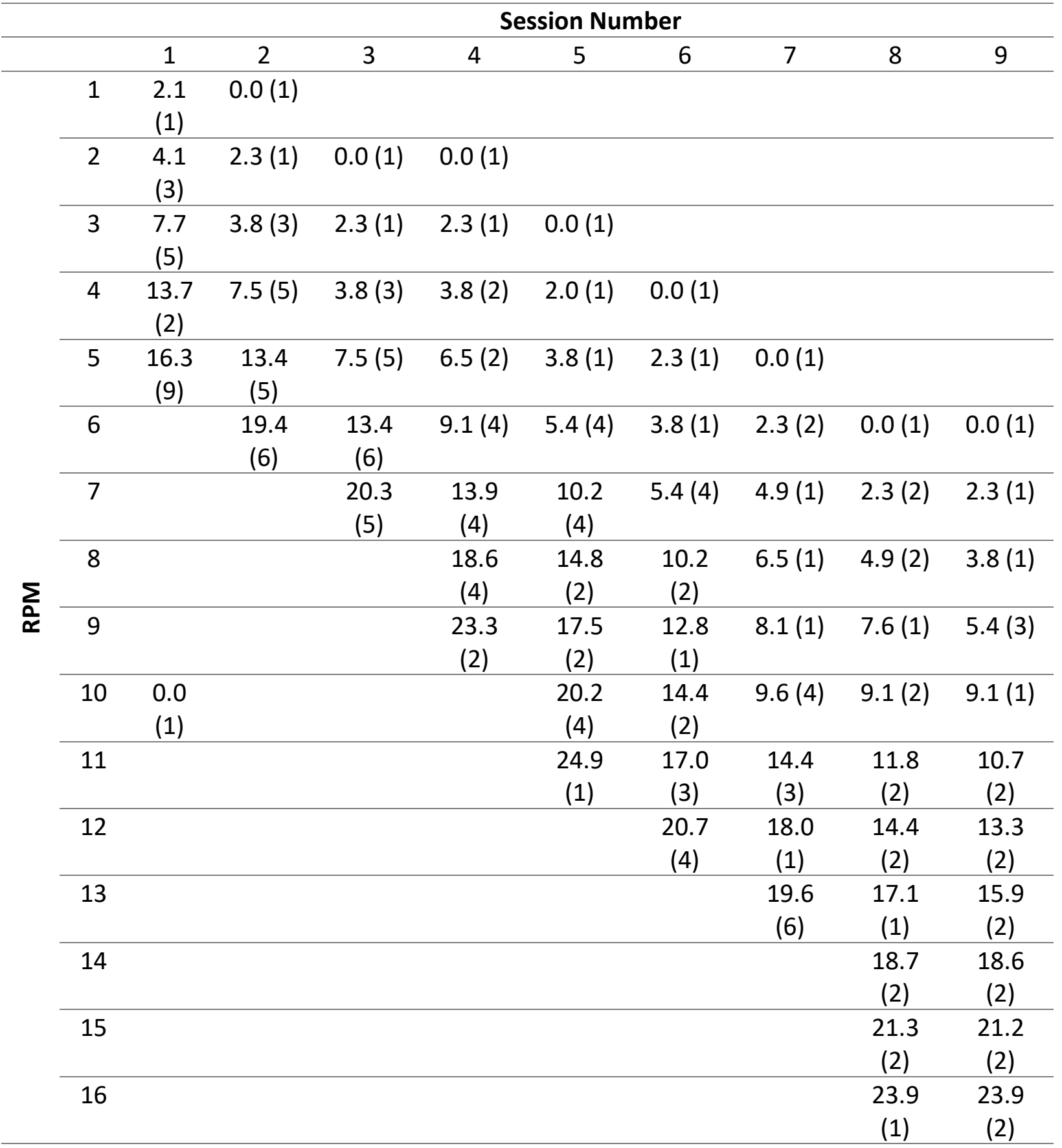

This table provides the detailed specifications of the standardized, incremental protocol, which was designed to match the median spin rate obtained from the 10 subjects in the personalized, incremental protocol [2]. An example staircase sequence and summary are shown graphically in Figure 1, but here we show exact details for replicability. Each of the 9 sessions are shown as columns. Each of the spin rates achieved throughout 
the protocol, in incremental units of 1 RPM, are shown as rows. In each cell is the time in minutes during the session at which that spin rate is incremented to, as well as (in parenthesis) the number of head tilt pairs which were performed at that spin rate. For example, on session 2 , the session began (time $=0.0$ minutes) by spinning up to 1 RPM and then performing 1 head tilt pair. After 2.3 minutes from the beginning of the session, the spin rate was incremented to 2 RPM and 1 head tilt pair was performed at that spin rate. Next (3.8 minutes into the session), the spin rate was incremented to 3 RPM, at which 3 head tilt pairs were performed. This sequence continued (down column 2) reaching 6 RPM after 19.4 minutes, where the subject performed six head tilt pairs. As another example, session 7 begins by immediately spinning up to 5 RPM for the first head tilt pair. Of note, session 1 begins (time $=0.0$ minutes) by spinning up to 10 RPM to perform 1 head tilt pair (for initial exposure to the CC illusion) and then slowing to 1 RPM (time=2.1 minutes) to begin the incrementally increasing staircase. 